IFUM 565-FT

hep-ph/9705233

\title{
Angular ordering and small-x structure functions.
}

\author{
Gavin P. Salam \\ INFN - Sezione di Milano, Via Celoria 16, 20133 Milano, Italy
}

\begin{abstract}
This talks examines the effect of angular ordering on the small- $x$ evolution of the unintegrated gluon distribution, and discusses the characteristic function for the CCFM equation.
\end{abstract}

For some time now it has been known that angular ordering [1] is an essential element in any description of small- $x$ final state properties [2]. As a first step of a programme to study the final state in small- $x$ physics, one should examine the effect of angular ordering on the small- $x$ evolution of the gluon structure function. Phenomenological studies have already been performed [3], but this talk will examine the solutions of the CCFM equation [2] from a more theoretical point of view.

The main difference between the BFKL [4] and CCFM equations is in the collinear region: in the BFKL case, the $i^{\text {th }}$ emission has transverse momentum $q_{i}>\mu$, with $\mu \rightarrow 0$; this regulates the collinear divergence which is present, but gives the wrong final state properties. In the CCFM case, angular ordering of emissions leads to the following condition (see figure 1):

$$
\theta_{i}>\theta_{i-1}, \quad \Rightarrow \quad q_{i}>z_{i-1} q_{i-1}
$$

with the corresponding gluon emission distribution being

$$
d \mathcal{P}_{i}=\frac{d^{2} q_{i}}{\pi q_{i}^{2}} d z_{i} \frac{\bar{\alpha}_{S}}{z_{i}} \Delta\left(z_{i}, q_{i}, k_{i}\right) \Theta\left(q_{i}-z_{i-1} q_{i-1}\right) .
$$

The non-Sudakov form factor $\Delta$, which is analogous to a probability for suppressing any further radiation, is defined by

$$
\ln \Delta\left(z_{i}, q_{i}, k_{i}\right)=-\int_{z_{i}}^{1} d z^{\prime} \frac{\bar{\alpha}_{S}}{z^{\prime}} \int \frac{d q^{\prime 2}}{q^{\prime 2}} \Theta\left(k_{i}-q^{\prime}\right) \Theta\left(q^{\prime}-z^{\prime} q_{i}\right) .
$$

(c) 1997 American Institute of Physics 


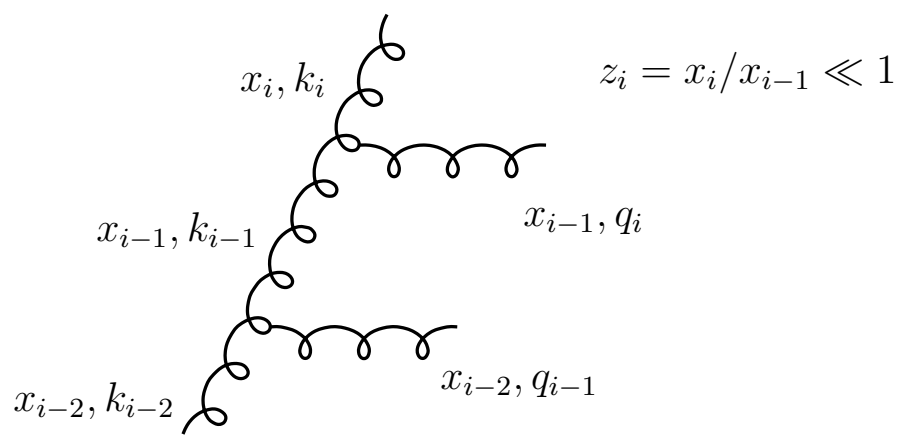

FIGURE 1. Labelling of momenta

The elimination of a large fraction of the small-transverse-momentum emissions means that angular ordering has a big effect on the final state. But in structure function evolution, since collinear singularities cancel, at leading order the BFKL and CCFM structure functions are equivalent.

As part of a program to carry out a full investigation of the effects of angular ordering at small $x$, this talk examines the component of the next-to-leading order corrections to structure function evolution that arise from angular ordering. Such effects are expected to be part of the full NLO contribution [5].

Qualitatively since angular ordering reduces the phase space for evolution, the exponent of the small- $x$ growth ought to be reduced. The symmetry, present in the BFKL equation, between large and small scales will be broken, favouring evolution to large momentum scales. Finally diffusion will be reduced because large jumps (down) in scale are suppressed.

There are two limits in which the effects of angular ordering should disappear: as $\alpha_{S} \rightarrow 0$, because the typical $z_{i-1} \sim \alpha_{S}$ will be very small (this justifies the assertion that for structure functions the effects of angular ordering are next to leading); and in the double-leading-logarithmic limit because the condition $q_{i}>q_{i-1}$ automatically satisfies the angular ordering condition.

The analytic treatment of the CCFM equation is more complicated than that of the BFKL equation because the gluon density contains one extra parameter, $p$, which defines the maximum angle for the emitted gluons. In DIS it enters through the angle of the quarks produced in the boson-gluon fusion. The equation for the CCFM density, $A(x, k, p)$, of gluons with longitudinal momentum fraction $x$ and transverse momentum $k$ is:

$\mathcal{A}(x, k, p)=\mathcal{A}^{(0)}(x, k, p)+\int \frac{d^{2} q}{\pi q^{2}} \frac{d z}{z} \frac{\bar{\alpha}_{S}}{z} \Delta(z, q, k) \Theta(p-z q) \mathcal{A}\left(x / z, k^{\prime}, q\right)$,

where $k^{\prime}=|\boldsymbol{k}+\boldsymbol{q}|$. By analogy to the BFKL equation one can develop some understanding of it by looking for eigensolutions (strictly speaking eigensolutions of the equation without an inhomogeneous term and with no upper limit 


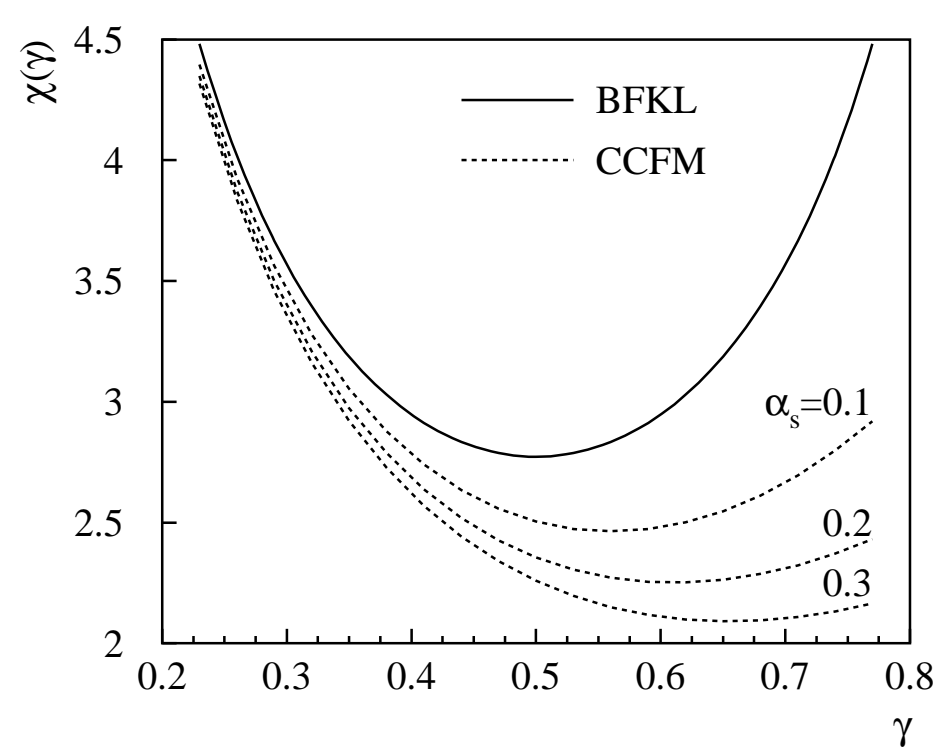

FIGURE 2. The BFKL and CCFM characteristic functions as a function of $\gamma$ for different values of $\alpha_{S}$.

in the $z$ integral) of the form

$$
x \mathcal{A}(x, k, p)=x^{-\omega} \frac{1}{k^{2}}\left(\frac{k^{2}}{k_{0}^{2}}\right)^{\gamma} G(p / k),
$$

where $G(p / k)$ parameterises the unknown dependence on $p$. For $0<\gamma<1$, one obtains a coupled pair of equations for $G$ and $\omega$ :

$$
p \partial_{p} G(p / k)=\bar{\alpha}_{S} \int_{p} \frac{d^{2} q}{\pi q^{2}}\left(\frac{p}{q}\right)^{\omega} \Delta(p / q, q, k) G\left(\frac{q}{k^{\prime}}\right)\left(\frac{k^{\prime 2}}{k^{2}}\right)^{\tilde{\gamma}-1},
$$

with the initial condition $G(\infty)=1$ and

$$
\omega=\alpha_{S} \tilde{\chi}\left(\tilde{\gamma}, \alpha_{S}\right)=\alpha_{S} \int \frac{d^{2} q}{\pi q^{2}}\left\{\left(\frac{k^{\prime 2}}{k^{2}}\right)^{\tilde{\gamma}-1} G\left(\frac{q}{k^{\prime}}\right)-\Theta(k-q) G(q / k)\right\} .
$$

In the second of these equations, if $G=1$ one notes that $\tilde{\chi}$ is just the BFKL characteristic function. Since $1-G(p / k)$ is formally of order $\alpha_{S}$, this demonstrates that angular ordering has a next-to-leading effect on structure function evolution. One can also show that in the limit of $\gamma \rightarrow 0$ the difference $\chi(\gamma)-\tilde{\chi}\left(\gamma, \alpha_{S}\right)$ tends to a constant, which implies corrections to the small- $x$ anomalous dimension of the form $\alpha_{S}^{3} / \omega^{2}$.

Though a number of asymptotic properties of $G(p / k)$ have been determined [6], it has not so far been possible to obtain its full analytic form. Further 
understanding requires numerical analysis. This has been carried out and figure 2 shows the results for $\tilde{\chi}$ compared to the BFKL characteristic function for three different values of $\alpha_{S}$. It illustrates that as $\alpha_{S} \rightarrow 0$ the two tend to coincide as happens also in the region $\gamma \rightarrow 0$ (the DLLA region).

The loss of symmetry under $\gamma \rightarrow 1-\gamma$ relates to the loss of symmetry between small and large scales. Indeed, in contrast to the BFKL case, there is no longer even a divergence at $\gamma=1$. Correspondingly, the minimum of the characteristic function gets shifted to the right and is lower.

Particularly strong is the change in the second derivative, which for $\alpha_{S}=0.2$ is reduced by a factor a two, indicating that for a given amount of diffusion to occur, one needs twice the rapidity ( $\ln x$ range) predicted by the BFKL equation, as seen for example in the result of Mueller [7] for the $x$ value where the operator-product expansion starts to break down due to diffusion,

$$
\ln \frac{x_{0}}{x} \simeq \frac{1}{2 \tilde{\chi}_{c}^{\prime \prime}} \ln \frac{Q^{2}}{\Lambda^{2}},
$$

where $\Lambda$ is the QCD scale, $x_{0}$ some starting point for the small- $x$ evolution, and $Q^{2}$ the hard scale of the problem.

\section{ACKNOWLEDGEMENTS}

This research was carried out in collaboration with G. Bottazzi, G. Marchesini and M. Scorletti and supported by funding from the Italian INFN. We are very grateful to M. Ciafaloni, Yu.L. Dokshitzer, A.H. Mueller and B.R. Webber for helpful discussions.

\section{REFERENCES}

1. Dokshitzer Yu.L., Khoze V.A., Troyan S.I., and Mueller A.H., Basics of Perturbative QCD, Editions Frontières, Paris, France 1991.

2. Ciafaloni M., Nucl. Phys. B296, 249 (1987); Catani S., Fiorani F., and Marchesini G., Phys. Lett. 234B, 339 (1990); Catani S., Fiorani F., and Marchesini G., Nucl. Phys. B336, 18 (1990).

3. Kwieciński J., Martin A.D., and Sutton P.J., Phys. Rev. D 53, 6094 (1996); Kwieciński J., Martin A.D., and Sutton P.J., Zeit. Phys. C71, 585 (1996).

4. Lipatov L.N., Sov. J. Phys. 23338 (1976); Kuraev E.A., Lipatov L.N., Fadin V.S., Sov. Phys. JETP 45199 (1977); Balitskii Ya., and Lipatov L.N., Sov. J. Nucl. Phys. 28822 (1978).

5. Fadin V.S., these proceedings.

6. Bottazzi G., Marchesini G., Salam G.P., and Scorletti M., hep-ph/9702418.

7. Mueller A.H., CU-TP-799, hep-ph/9612251. 